\title{
Costo efectividad de un programa de rehabilitación cardiovascular para personas post infarto agudo al miocardio: un análisis teórico
}

\author{
José Miguel Gómez ${ }^{1}$, Jame Rebolledo ${ }^{1}$, Paulina López ${ }^{2}$, Marcelo Cano ${ }^{1}$. \\ 1. Kinesiólogo, Departamento de Kinesiología, Facultad de Medicina. Universidad de Chile. \\ 2. Escuela de Kinesiología, Universidad de Chile.
}

Introducción: Pese a que la efectividad de los programas de prevención secundaria en pacientes con enfermedades cardiovasculares (ECV) ha sido ampliamente demostrada, su implementación tanto Chile como a nivel internacional ha sido pobre.

Objetivo: Determinar la tasa costo-efectividad de una propuesta teórica de Programa de Rehabilitación cardiovascular (PRC) basada en la evidencia y validada por expertos, en personas post Infarto Agudo al Miocardio (IAM) en el nivel secundario de atención en salud, pertenecientes al Servicio de Salud Metropolitano Norte, Región Metropolitana.

Método: Se elaboró un protocolo teórico de un PRC integral basado en las guías clínicas internacionales (AHA, AACVPD, NICE, ESC, NHMRC, Victoria), ajustado a la realidad chilena, el cual fue costeado. Luego se estimó los años de vida por muerte prematura con y sin participación en un PRC, para obtener finalmente los Años de Vida Ganados (AVG). Con ello se calculó la tasa de costo-efectividad.

Resultados: El costo anual del centro de rehabilitación cardíaca es de \$64.407.065 CLP. La Razón Incremental de Costo Efectividad (ICER), considerando una reducción de la mortalidad tardía del 25\%, es de CLP\$475.209,72/AVG. Valor que al ser menor al Producto Interno Bruto per cápita, se considera como una intervención muy costo efectiva.

Conclusión: Un programa de rehabilitación cardiaca integral post-IAM parece muy costo-efectivo. 


\section{Cardiovascular rehabilitation program in post myocardial infarction: a theoretical analysis of cost effectiveness}

Background: Although the effectiveness of secondary prevention programs in patients with cardiovascular disease (CVD) has been widely demonstrated, its implementation both in Chile and other countries has been scarce.

Aim: To determine the cost-effectiveness of an evidence-based theoretical comprehensive cardiac rehabilitation (CCR), validated by experts, for post-acute myocardial infarction (AMI) patients at the secondary level from the Chilean Public Health System.

Methods: A theoretical protocol of a CCR program based on recommendations from international guidelines (AHA, AACVPD, NICE, ESC, NHMRC, Victoria) was elaborated and adjusted to the Chilean conditions. A cost analysis was performed. Life years due to premature death were estimated with and without participation in the cardiac rehabilitation program (CRP). We obtained gained life-years, and calculated the ratio of cost-effectiveness.

Results: The annual cost of the cardiac rehabilitation center is $\$ 64,407,065$ CLP. The Incremental Cost Effectiveness Ratio (ICER) considering a reduction in late mortality of $25 \%$, is CLP\$475.209,72/ AVG. Since it is less than one per capita gross domestic product, it is considered as a very cost-effective intervention.

Conclusion: A comprehensive cardiac rehabilitation program post AMI is very cost-effective for use in a in public health service. It should be considered to review Optimal frequency and intensity of exercise in order to achieve optimal results should be determined.

Keywords: myocardial infarction; rehabilitation, cardiovascular; cost-effectiveness 


\section{Introducción}

Los programas de rehabilitación cardíaca (PRC) secundaria de enfoque multidisciplinario han demostrado su efectividad en el cuidado integral de las personas con patología cardiovascular ${ }^{1,2}$, reduciendo la mortalidad cardiovascular, disminuyendo la cantidad de rehospitalizaciones y mejorando la calidad de vida de los personas. ${ }^{3}$ En el año 2011 la tasa de mortalidad por enfermedad isquémica del corazón (IAM) era de 41,7 por 100.000 habitantes, con 7.197 defunciones a nivel naciona ${ }^{14}$. La alta recurrencia de personas que sobreviven a un IAM, determina un gran número de personas con alto riesgo cardiovascular, las que de no ser controladas adecuadamente, significarán una elevada carga financiera para el sistema de salud 5 . Datos obtenidos en Estados Unidos han mostrado que, independiente de la edad, durante el primer año post IAM muere un $26 \%$ de las mujeres y un $19 \%$ de los hombres, mientras que durante los primeros cinco años fallecerá un $47 \%$ de las mujeres y un $36 \%$ de los hombres ${ }^{6}$.

El PRC proporciona una oportunidad altamente costo-efectiva para educar a las personas después de un episodio mórbido coronario y permitir controlar los principales factores de riesgo cardiovascular ${ }^{2}$. Para pacientes post IAM se recomienda una prescripción de ejercicio físico basada en una evaluación específica de la condición física. La prescripción debe incluir frecuencia de sesiones, intensidad de los ejercicios, duración de cada uno de ellos, modalidad (entrenamiento para mejorar la capacidad cardiorespiratoria y/o fuerza muscular) y la modalidad de progresión ${ }^{1}$. Pese a que el riesgo de complicaciones cardiovasculares aumenta transitoriamente durante el ejercicio vigoroso e infrecuente, en especial para personas que tienen enfermedad coronaria y son sedentarias $^{7}$, el ejercicio físico, considerado una importante herramienta terapéutica, debe estar a cargo de un equipo con formación en rehabilitación cardiovascular y con preparación para asumir una emergencia ${ }^{2,8,9}$.

Desde el año 2005, el diagnóstico, tratamiento y seguimiento del infarto agudo al miocardio es parte de las Garantías Explícitas en Salud. Se incorpora la garantía de acceso a prevención secundaria, lo que incluye profilaxis farmacológica; hipolipemiante, educación sobre hábitos de vida saludables, control adecuado de la hipertensión arterial y la diabetes. Sin embargo, a pesar de la evidencia disponible, la garantía de seguimiento no explicitó el acceso a un programa de ejercicio supervisado ${ }^{10}$.

En nuestro país, existen PRC basados en ejercicio físico realizados en su mayoría en instituciones privadas y con- centrados en la Región Metropolitana ${ }^{11}$. Las razones de la falta de implementación de dichos programas podrían radicar en la falta de personal entrenado, limitaciones económicas, ausencia de espacio físico y en la baja percepción del beneficio clínico o rentabilidad que se puede obtener de un $\mathrm{PRC}^{12}$. Sin embargo, nuestro grupo de investigación recientemente publicó un estudio de evaluación económica en este ámbito ${ }^{13}$, concordando con lo publicado previamente en otros estudios internacionales, que señalan que la implementación de un PRC es costo-efectivo $2,7,14$ y 15 .

El propósito de la presente publicación es estimar la tasa de costo-efectividad de una propuesta teórica de PRC basada en la evidencia y validada por expertos, que incluye ejercicio físico y consejerías, entregando antecedentes que permitan su implementación de manera integral en el Sistema de Salud público chileno.

\section{Método}

En la propuesta ya publicada ${ }^{13}$ se realizó un estudio de costo-efectividad, considerando las recomendaciones de la Guía de Evaluación Económica del MINSAL $(2013)^{16}$. Las alternativas que se compararon fueron a) Trombolisis tras un IAM y b) Trombolisis más incorporación a un PRC.

Se propuso en ese artículo un PRC según recomendaciones de la American Heart Association, la Heart Foundation (Australia), la European Society of Cardiology y el National Institute for Health and Clinical Excellence (NICE). Este programa fue realizado en base a un protocolo ambulatorio supervisado, técnicamente viable, ajustado a la realidad chilena y aprobado por un comité de expertos compuesto por kinesiólogos expertos en rehabilitación cardiometabólica y fisiología del ejercicio.

Para ajustar el programa a la población objetivo se estimó la demanda anual de personas sobrevivientes a un IAM en el Servicio de Salud Metropolitano Norte (SSMN), con una población compuesta por personas mayores de 50 años, de predominio masculino, que presentan uno o más factores de riesgo cardiovascular y enfermedades metabólicas.

En el estudio realizado ${ }^{13}$, se consideró un horizonte temporal de un año para los costos, para dar cobertura a la demanda anual estimada y en el caso de los efectos, se consideró la sobrevida a 24 meses tras el PRC ${ }^{17}$. Fueron considerados como gastos directos del PRC el recurso humano y materiales audiovisuales y, como gastos indirectos, la infraestructura, equipamiento, servicios básicos y gastos administrativos. Los activos fueron valorados 
según mercado, calculando el costo anual según los años de vida útil definidos por el Servicio de Impuestos Internos.

El recurso humano necesario para implementar el PRC estaba conformado por un médico con una jornada de 11 h/ semana, dos kinesiólogos (as) (una jornada $33 \mathrm{~h} / \mathrm{se}$ mana y una jornada de $22 \mathrm{~h} /$ semana), dos enfermeros (as) (una jornada $33 \mathrm{~h} /$ semana y una jornada de $22 \mathrm{~h} /$ semana), un nutricionista ( $2 \mathrm{~h} /$ semana) y un psicólogo clínico con una jornada de $11 \mathrm{~h} /$ semana. Sus remuneraciones fueron estimadas según la ley 18.834 para profesionales de categoría B y la ley 19.664 para los de categoría A.

La propuesta de PRC estaba compuesta por tres sesiones semanales de entrenamiento de la capacidad cardiorrespiratoria, donde la intensidad era 50-70\% de la frecuencia cardíaca máxima obtenida en el test de esfuerzo durante las 3 primeras semanas y de $70-80 \%$ en las últimas 5 semanas del programa, con una duración de $60 \mathrm{~min}$, aproximadamente. Por otra parte con el objetivo de aumentar la fuerza muscular, en el PRC dos veces por semana se propuso realizar ejercicios con 12 a 15 repeticiones a un nivel de esfuerzo de fatiga moderada, 1-3 series de 8-10 ejercicios de grupos musculares de extremidades superiores e inferiores.

El PRC, además, tenía 9 talleres grupales orientados hacia la modificación de estilos de vida/hábitos y control del estrés, 1 taller con enfoque de género (exclusivo para mujeres) y 2 consejerías individuales.

Para estimar la eficacia en la reducción de la mortalidad, primero se estimaron los años perdidos por muerte prematura (AVPMP) basados en la estadística nacional de mortalidad tardía post-trombolisis. La reducción de la mortalidad tardía asociada al PRC, nos permitió estimar los Años de Vida Ganados (AVG) secundarios a la intervención, como indicador unidimensional de efectividad. La relación entre AVG y los costos diferenciales se utilizó para estimar la Razón de Costo Efectividad Incremental (RCEI o ICER en inglés).

El índice de costo-efectividad, fue clasificado según las recomendaciones que el MINSAL realiza para este tipo de estudios ${ }^{16}$. Si bien esta clasificación se utiliza para estudios que expresan sus resultados en indicadores multidimensionales, como los AVAC (años de vida ajustados por calidad) y AVAD (años de vida ajustados por discapacidad), consideramos que el PIB per cápita es un umbral aceptable para AVG.

Se analizó la estimación de la demanda mediante el análisis bivariable de la incidencia y la letalidad del IAM. Los valores de variabilidad consideran las tendencias de incidencia y letalidad a 6 años ${ }^{18}$. Se efectuó un análisis univariable de los cambios de incidencia, letalidad, derivación y eficacia del PRC y su impacto sobre los resultados (AVG e ICER).

Finalmente, se realizó un Análisis de Sensibilidad de tres escenarios, que considera: 1) menor derivación documentada $(15 \%)$ y adherencia de $50 \% ; 2$ ) derivación y adherencia de $50 \%$ y 3 ) derivación $50 \%$ y adherencia $100 \%$.

\section{Resultados}

Dado que la alternativa B incluye la alternativa A (trombolisis post IAM), se realizó un costeo incremental, es decir, sólo considera la diferencia atribuida a la implementación de un PRC. Así, el costo anual de implementación del PRC, incluyendo la infraestructura para funcionar a capacidad máxima, fue calculado en $\$ 64.407 .065$ CLP. Mientras que de los 218 pacientes que ingresaron con diagnóstico de IAM se estima que habrían sobrevivido 112. Por lo que el costo por persona atendida es de $\$ 575.063$ CLP.

Con base en los datos de mortalidad asociada a IAM descritos en el Reporte de Vigilancia de Enfermedades No Transmisibles (MINSAL 2011) y la tasa de mortalidad tardía ( 2 años) tras una trombolisis ${ }^{19}$, se estimó que la suma de AVPMP para ambos géneros fue de 558,4.

Dado que los estudios avalan que un PRC puede disminuir la mortalidad tardía hasta en un $25 \%$, la suma de los AVPMP de ambos sexos caería a 418,8. O visto desde otra perspectiva, se ganan 135,53 años (AVG) por haber participado en el PRC respecto a la expectativa de sobrevida que habrían tenido las personas tratadas exclusivamente con un tratamiento trombolítico tradicional. Este cambio sería consecuencia de las mejoras en la condición física y cambios en los estilos de vida estimados con base en las publicaciones previas.

Así la relación entre el costo incremental de un PRC y los AVG nos entrega un ICER de \$475.209 CLP. En un plano cartesiano de costo efectividad (Figura 1), el ICER obtenido se posiciona en el cuadrante superior derecho, donde con mayores costos hay mayor efectividad, por lo tanto, la elección de implementar un PRC dependerá de la máxima disposición a pagar (o aceptar) por los efectos en la sobrevida. Usando el PIB per cápita como umbral de referencia, el ICER es menor al PIB per cápita 2013 (USD\$ 15.764 según datos de Banco Mundial), por lo cual la intervención parece ser muy costo efectiva. Según un estudio nacional, la incidencia en 6 años, aumentó 9,2\% en el grupo entre 55 y 64 años, mientras que el grupo entre 65 y 74 años disminuyó 11,3\%. Se 


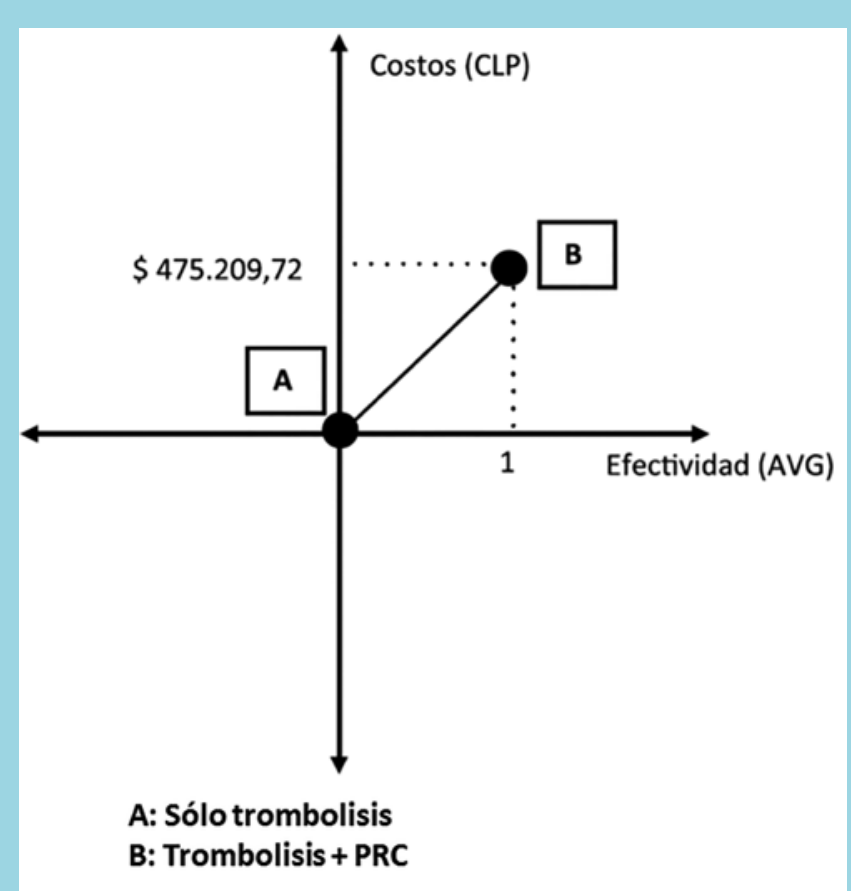

Figura 1: Gráfico de la razón costo efectividad incremental (ICER en el plano carteriano. La pendiente entre A y $B$ corresponde al ICER.

consideró un cambio anual proporcional. En cuanto a la letalidad, disminuyó $0,7 \%$ por año considerando todos los grupos etarios18. Con base en estos datos se consideró que la máxima demanda anual fluctuaría entre 95 y 122, valores menores que la capacidad máxima estimada para el programa modelo (260 personas). Sin embargo, si incidencia y letalidad cambiaran simultáneamente, la demanda (291 personas) sobrepasaría la capacidad total del centro al sexto año.

El Análisis de Sensibilidad Univariable consideró la variabilidad de los parámetros incidencia, letalidad, derivación y eficacia. De ellos el factor más influyente es el cambio en la derivación, estimando una efectividad de 54,21 AVG (40\% de derivación) y de ${ }^{20,32}$ AVG (15\% de derivación). De acuerdo a estos datos, el ICER en el peor de los casos alcanzaría un valor de \$3.168.141,5/ AVG. Esto corresponde a 40,66\% del PIB per cápita del 2013 lo que continúa siendo muy costo efectivo.

Finalmente, en el mejor de los escenarios, vale decir un $100 \%$ de derivación y un $50 \%$ de adherencia, los AVG disminuyen a 67,5 AVG, con una ICER de \$954.522/ AVG, lo que corresponde a $12,25 \%$ del PIB per cápita 2013. Pero si la derivación fuera de sólo un $15 \%$ y la adherencia se mantuviera en el 50\%, los AVG obtenidos sólo serían de 10,1 AVG, con un ICER de \$6.636.480/
AVG, lo que representa 81,66\% del PIB per cápita 2013.

\section{Discusión}

En un escenario donde los seguros de salud siguen mostrando una tendencia a pagar por prestación y los esfuerzos por contener costos parecieran insuficientes para detener la tendencia creciente del gasto en salud, se hace necesario que las intervenciones en salud sean costo-efectivas, en especial si el foco de ellas es la prevención.

Los resultados de este estudio señalan que el costo de sumar una año de vida alcanza aproximadamente el $6 \%$ del PIB per cápita chileno según fuentes del Banco Mundial del año 2013, lo que significa que el ICER de un PRC basado en el evidencia disponible parece ser ampliamente costo-efectivo para el país. Resultados que se mantendrían pese a que la derivación cayera desde $20 \%$ (datos sin publicar) al $15 \%$, en cuyo caso el ICER alcanzaría un 40,7\% del PIB per cápita.

Este estudio sólo consideró el impacto de la mortalidad en la estimación de los AVG. Sin embargo, la evidencia disponible avala que los PRC además tienen un impacto positivo sobre la calidad de vida, la capacidad aeróbica máxima y los factores de riesgos asociados a la enfermedad coronaria ${ }^{20,21}$, el cual puede ser objetivado por medio de estudios que cuantifiquen o estimen los Años de Vida Ajustados por Calidad (QALY) y/o Años de Vida Ajustado por Discapacidad (DALY), beneficios que sin duda podrían sumarse a los AVG revelados por el presente estudio.

Consideramos que al incluir en los costos netos (diferencia entre gastos y ahorros/ganancias) sólo aquellos asociados al PRC, excluyendo el ahorro atribuible a la disminución de la tasa de re-infarto y la hospitalización subsequente $^{22,23}$, los costos totales podrían estar sobreestimados.

Dado que los Objetivos Sanitarios vigentes contemplan un aumento de un $10 \%$ de la sobrevida al primer año del IAM, consideramos que a la luz de la evidencia y de los resultados de este estudio, pareciera recomendable la incorporación de manera explícita de un PRC de enfoque integral en la Guías Clínicas, de manera de sumar el ejercicio físico como una herramienta terapéutica en el tratamiento de la enfermedad coronaria y en la prevención del re-infarto, apalancando así el cumplimiento de esta meta. Finalmente, la evidencia apunta a que grupos de personas con un perfil de riesgo mayor pueden obtener mayor beneficio de un $\mathrm{PRC}^{24}$, así individuos con hipertensión arterial y diabetes tipo II podrían beneficiarse de forma 
significativa $^{25}$. Es necesario desarrollar nuevos estudios con pacientes que determinen la efectividad y costos asociados, tanto desde la perspectiva del sistema de salud como de los usuarios/as y sus familias. Por lo tanto, sugerimos la implementación de un programa piloto.

\section{Agradecimientos}

A los kinesiólogos que participaron en la revisión y corrección del programa modelo: Claudia Román, Klga.
Especialista Cardiovascular acreditada DENAKE, Complejo Hospitalario San José; Manuel Alvear, Klgo. Especialista Fisiología Aeroespacial, Universidad del Desarrollo y Karen Rouliez, Klga. Programa de Rehabilitación Cardiaca HCUCH, y docente del Diplomado en Kinesiología Cardiorespiratoria. Y a los consultores del área economía; Sylvia Galleguillos, Directora IAS Facultad de Economía y Negocios, Universidad de Chile, y Marianela Castillo, Economista MINSAL.

\section{Referencias}

1. BALADY G, WILLIAMS M, ADES P, BITTNER V, COMOSS P, FOODY J, et al. Core components of cardiac rehabilitation/secondary prevention programs: 2007 update: a scientific statement from the American Heart Association Exercise, Cardiac Rehabilitation, and Prevention Committee, the Council on Clinical Cardiology; the Councils o. Circulation 2007; 115: 2675-82

2. ROMERO T. La rehabilitación cardíaca como punto de partida en la prevención secundaria de la enfermedad coronaria. Rev Med Chile [revista en la Internet]. 2000 agosto. Citado el 31 de marzo de 2013; 128: 923-34.

3. ANDERSON L, OLDRIDGE N, THOMPSON DR, ZWISLER AD, REES K, MARTIN N, et al. Exercise-Based Cardiac Rehabilitation for Coronary Heart Disease: Cochrane Systematic Review and Meta-Analysis. J Am Coll Cardiol. 2016; 67: $1-12$.

4. DEIS. Serie de Mortalidad por causas 2000-2010. Actualizado el 2012 Ministerio de Salud, Secretaría de Salud Pública. Reporte de Vigilancia de Enfermedades No Transmisibles (ENT). Recuperado septiembre 2012. Disponible en: http://epi.minsal.cl/epi/0notransmisibles/vent/VENT_Mercosur_FINAL_17092011.pdf
5. MEHTA LS, BECKIE TM, DEVON HA, GRINES CL, KRUMHOLZ HM, JOHNSON MN, et al. Acute myocardial infarction in women: A scientific statement from the American Heart Association. Circulation 2016; 133: 916-47.

6. YU CH-M, LAU CH-P, CHAU J, MCGHEE S, KONG S-L, MAN-YUNG CHEUNG B, et al. A Short Course of Cardiac Rehabilitation Program is Higly Cost Effective in Improving Long-Term Quality of Life in Patients With Recent Myocardial Infarction or Percutaneous Coronary Intervention. Archives of Physical Medicine and Rehabilitation, 2004; 85: 1915-22.

7. FLETCHER G, BALADY G, EZRA A, CHAITMAN B. Excercise Standards for testing and training: A Statement for Healthcare Professionals from the American Heart Association. Circulation, 2011; 104, 1.694-1.740.

8. HUNT SH, BAKER D, CHIN M, CINQUEGRANI M, FELDMAN A. ACC/AHA Guidelines for evaluation and management of chronic heart failure in adult. A report of the American College of Cardiology / American Heart Asociation Task Force on Practice Guidelines. Circulation, 2001; 104, 2.996-3.007. 
9. MINISTERIO DE SALUD, SECRETARÍA DE SALUD PÚBLICA. Reporte de Vigilancia de Enfermedades No Transmisibles (ENT). Recuperado Noviembre 2016. Disponible en: http://www.bibliotecaminsal.cl/wp/wp-content/ uploads/2016/04/Infarto-Agudo-del-Miocardio.pdf

10. SANTIBÁÑEZ C, PÉREZ-TÉRZIC C, LÓPEZ-JIMÉNEZ F, CORTÉS-BERGODERI M, ARAYA MV, BURDIAT G. Situación actual de la rehabilitación cardiaca en Chile, Rev Med Chile 2012; 140: 561-568.

11. KORENFELD Y, MENDOZA-BASTIDAS C, SAAVEDRA L, MONTERO-GÓMEZ A, PEREZ-TERZIC C, THOMAS $\mathrm{RJ}$, et al. Current status of cardiac rehabilitation in Latin America and the Caribbean. Am Heart J. 2009; 158: 480-7.

12. LOPEZ P, REBOLLEDO J, GÓMEZ J. Costo efectividad de un programa de rehabilitación cardiovascular modelo para personas post infarto agudo al miocardio en el Servicio de Salud Metropolitano Norte. Rev Med Chile 2016; 144: 456 - 464.

13. TAYLOR R, WATT A, DALAL H, EVANS P, CAMPBELL $\mathrm{J}$, READ K, et al. Home-based cardiac rehabilitation versus hospital-based rehabilitation: A cost effectiveness analysis. International Journal of Cardiology, 2007; 119: 196-201.

14. BRIFFA T, ECKERMAN S, GRIFFITHS A, HARRIS P, HEALTH M, FREEMAN S, DONALDSON L, et al. Cost-effectiveness of rehabilitation after an acute coronary event: a randomisedcontroled trial. Medical Journal of Australia, 2005; 183: 450-455

15. Ministerio de Salud. Guía metodológica para la Evaluación Económica de Intervenciones en Salud en Chile, 2013.

16. LAWLER P, FILION K, EISENBERG M. Efficacy of exercise-based cardiac rehabilitation post-myocardial infarction: A systematic review and meta-analysis of randomized controlled trials. Am Heart J, 2011; 162: 571-584.
17. NAZZAL C. Y ALONSO F. Incidencia y letalidad por Infarto Agudo al Miocardio en Chile: 2001 - 2007. RevMed Chile 2011; 139: 1253-1260.

18. MARTÍNEZ A, NAZZAL C, FAJURI N, BARRA L, MAYERSON A, CAVADA G, et al. Mortalidad post infarto del miocardio en Chile: Comparación de los registros de angioplastia primaria versus trombolisis. Rev Chil Cardiol 2010; 29: 29-36.

19. COLIN W, ALISON E. Cardiac rehabilitation and quality of life: A systematic review. International Journal of NursingStudies 2012; 49: 755-771.

20. GRIFFO R, AMBROSSETTI M, TRAMARIN R, FATTIROLLI F, TEMPORELLI P, VESTRI A, et al Effective secondary prevention through cardiac rehabilitation after coronary revascularization and predictors of poor adherence to lifestyle modification and medication. Results of the ICAROS Survey. International Journal of Cardiology (2012/ in press)

21. ALVEAR S, CANTEROS J, RODRÍGUEZ P. Estudio retrospectivo de costos de tratamientos intensivos por paciente y día cama. RevMed Chile 2010; 138: 558-566

22. ALVEAR S, CANTEROS J, JARA J, RODRÍGUEZ P. Costos reales de tratamientos intensivos por paciente y día cama. RevMed Chile 2013; 141: 202-208

23. SCHWAAB B, WALDMANN A, KATALINIC A, SHEIKHZADEH A, RASPE H. In-patient cardiac rehabilitation versus medical care - a prospective multicenter controlled 12 months follow-up in patients with coronary heart disease. Eur J Cardiovasc Prev Rehabil. 2011; 18:581-6.

24. O'CONNOR C., WHELLAN D., LEE K., KETEYIAN S., COOPER L., ELLIS S., et al. Efficacy and Safety of Exercise Training in Patients With Chronic Heart Failure HF-ACTION Randomized Controlled Trial. JAMA. 2009; 301:1439-1450. 